\title{
Perspetivas futuras na monitorização do ritmo cardíaco e a utilização de smartwatch
}

Carolina Araújo Soares, ${ }^{1}$ Andreia Figueira Pinto, ${ }^{2}$ Raquel Andrade, ${ }^{3}$ Ricardo C. Rodrigues ${ }^{4}$

\section{RESUMO}

A crescente utilização de smartwatches passou a fazer parte do quotidiano dos seus utilizadores, com especial foco na atividade física, ao permitir monitorizar o exercício realizado em termos de intensidade, duração, gasto energético e, mais recentemente, monitorização da frequência cardíaca. A potencial utilização destes dispositivos eletrónicos no campo da medicina é um marco importante, permitindo, através da monitorização do ritmo cardíaco, a possível deteção de períodos de arritmia, como a fibrilação auricular, com extensão à monitorização de arritmias ventriculares potencialmente fatais, como o prolongamento do intervalo QT, este último com particular utilidade na monitorização à distância durante o período da pandemia COVID-19. Os principais estudos apresentados apresentam várias limitações; no entanto, elucidam os leitores para a importância da crescente evolução tecnológica e para o seu potencial impacto prognóstico nos utilizadores destes dispositivos.

Palavras-chave: Smartwatch; Monitorização remota; Fibrilação auricular; Arritmias.

4 tecnologia é uma área em constante desenvolvimento e com grande potencial, tendo conquistado progressivamente o seu lugar no nosso dia-a-dia, funcionando como facilitador nas mais variadas vertentes da vida diária, desde a comunicação ao acesso fácil e rápido a grande quantidade de informação praticamente ilimitada. Também a medicina beneficia da expansão da indústria tecnológica, não só pelo acesso privilegiado e facilitado a informação científica de qualidade, mas também na área da investigação clínica, desde a fisiopatologia a novas abordagens diagnósticas terapêuticas, entre outras.

Com o advento dos smartwatches e com a sua utilização crescente, a tecnologia passou a fazer parte integrante da atividade física, permitindo monitorizar o

1. Centro de Saúde do Bom Jesus. Funchal, Região Autónoma da Madeira, Portugal. 2. Centro de Saúde de Santo António. Funchal, Região Autónoma da Madeira, Portugal.

3. Centro de Saúde do Caniço. Caniço, Região Autónoma da Madeira, Portugal. 4. Serviço de Cardiologia, Hospital Dr. Nélio Mendonça. Funchal, Região Autónoma da Madeira, Portugal. exercício realizado em termos de intensidade, duração e de gasto energético, por exemplo. Mais recentemente, estes dispositivos foram equipados com sensores que permitem também a monitorização da frequência e ritmo cardíacos, abrindo-se a janela para a sua utilização na deteção de alterações do ritmo cardíaco, nomeadamente a fibrilação auricular (FA). Esta é a arritmia mais frequente, sendo uma importante causa de morbimortalidade, sobretudo devido ao risco de acidente vascular cerebral (AVC) que lhe está associado.

De acordo com os dados do estudo Safira, a prevalência global de FA na população portuguesa com mais de 65 anos é de 9\%. Existem vários fatores de risco que lhe estão associados, sendo a idade e a hipertensão arterial os fatores com maior peso no desenvolvimento desta entidade. Dada a forte associação entre o desenvolvimento de FA e a idade é expectável que a sua incidência e prevalência aumente nas próximas décadas, devido ao envelhecimento populacional, o que reforça a necessidade de desenvolvimento de estratégias que 
permitam a deteção precoce desta arritmia, principalmente episódios em indivíduos assintomáticos e durante fenómenos paroxísticos, permitindo a intervenção atempada de modo a prevenir a ocorrência de eventos tromboembólicos e suas consequências. ${ }^{1}$

A FA pode ser classificada em vários tipos; quando se trata de uma FA paroxística, a sua natureza pode condicionar o atraso no diagnóstico de FA através do eletrocardiograma (ECG). Neste contexto, Perez e seus colaboradores desenvolveram o Apple Heart Study, cujos resultados foram publicados em novembro de 2019. Este é um estudo de coorte prospetivo, open-label, constituído por um único grupo de participantes, selecionado de acordo com critérios de elegibilidade definidos, de entre os quais se destaca a idade igual ou superior a 22 anos e a posse de iPhone ou Apple Watch. ${ }^{2}$

O objetivo primário do estudo era a notificação após a deteção de pulso irregular durante um período superior a 30 segundos, confirmado posteriormente com um patch de monitorização eletrocardiográfica, enviado para o domicílio dos indivíduos notificados, que deveria ser utilizado durante sete dias, com o intuito de confirmar a existência de FA. ${ }^{2}$

Num universo de 419.927 participantes, apenas $24.626(5,9 \%)$ apresentavam idade superior ou igual a 65 anos. Da totalidade dos participantes receberam a notificação de pulso irregular 2.161 (0,52\%) participantes, sendo que $3,2 \%$ apresentavam uma idade igual ou superior a 65 anos. ${ }^{2}$

Apenas $0,16 \%$ dos participantes com idade inferior a 40 anos foram notificados, sendo que neste grupo a taxa de concordância com confirmação de FA em monitorização prolongada foi mais baixa (18\%) do que nos restantes, traduzindo a história natural da FA, com o predomínio de FA paroxística nos estádios iniciais da doença. A taxa global de deteção de FA na monitorização prolongada de doentes que receberam notificação foi de $34 \%$, sendo clinicamente relevante pois $89 \%$ tinham períodos em FA superiores a 60 minutos, condicionando uma carga arrítmica importante. ${ }^{2}$

Os resultados têm uma validade ambígua. Apesar da probabilidade de notificação de pulso irregular ser baixa, e de apenas $33 \%$ dos participantes notificados utilizarem o patch, os dados obtidos foram concordantes com a presença de FA em cerca de $84 \%$ dos casos que usaram o patch de monitorização e o devolveram. Sete participantes notificados $(0,8 \%)$ e 321 não notificados $(0,1 \%)$ receberam o diagnóstico de AVC durante o período do estudo. ${ }^{2}$

Foram assinaladas várias limitações pelos autores, desde o perfil dos participantes com destaque para a idade, o facto de serem exclusivamente utilizadores de iPhone e Apple Watch e à perda de follow-up. Os autores referem que o estudo não apresentava como objetivo avaliar a sensibilidade, especificidade ou falsos positivos do algoritmo nem avaliá-lo como método de rastreio de FA, pelo que apenas participantes com probabilidade de elevada carga arrítmica foram notificados. No entanto, concluíram que os dados obtidos corroboravam a hipótese de o algoritmo detetar corretamente FA e permitir notificar os participantes de forma a ser efetuado estudo adicional. A ausência de notificação não exclui a possibilidade de diagnóstico de FA. ${ }^{2}$

Após a publicação do Apple Heart Study foram várias as críticas e comentários aos seus resultados. Segundo Campion e seus colaboradores, a importância do estudo não está na validade do algoritmo per si, mas no que este estudo representa: a evolução tecnológica em que um smartwatch tem a capacidade de detetar arritmias que justifiquem avaliação e tratamento médico precoce. $^{3-4}$

Atualmente, a aplicação Apple Watch ECG está aprovada pela US Food and Drugs Administration (FDA) e classificada como dispositivo de classe II apenas na deteção de FA. Não está recomendada na deteção de outras arritmias. ${ }^{5}$

$\mathrm{Na}$ atual temática da pandemia que abalou o mundo, a utilização dos smartwatches na monitorização do ritmo cardíaco mostrou a sua relevância ao proporcionar a possibilidade do acompanhamento à distância. Perante a inexistência de um tratamento eficaz para a COVID-19, foram várias as terapêuticas propostas e utilizadas, numa perspetiva de controlo sintomático, curativo ou com impacto prognóstico na morbimortalidade. Destas, a associação de hidroxicloroquina e azitromicina foi uma das terapêuticas mais utilizadas, mas também uma das que mais preocupações gerou entre os clínicos ao considerar complicações graves que podem advir da sua utilização, nomeadamente o potencial arritmogénico caracterizado pelo prolongamento do intervalo QT. Deste modo, foram estabelecidas estratégias de monitorização do intervalo QT. ${ }^{6}$ 
Neste contexto, Strik e seus colaboradores desenvolveram um estudo que tinha por objetivo a validação da Apple Watch ECG na medição do intervalo QT, com recurso a posições alternativas à derivação DI (punho esquerdo), nomeadamente no tornozelo esquerdo e na grelha costal esquerda. ${ }^{6}$

Foi avaliada uma população de 100 indivíduos com ECG em ritmo sinusal, com predomínio do sexo masculino (59\%), com uma média de idades de $67 \pm 7$ anos, sendo que $35 \%$ apresentava diagnóstico prévio de patologia cardíaca. Oito indivíduos foram identificados com sendo de alto risco, todos através do smartwatch. O estudo concluiu que a aplicação permitiu a monitorização adequada do intervalo QT sempre que o smartwatch era utilizado no punho esquerdo em $85 \%$ dos indivíduos e em $94 \%$ dos casos quando utilizadas as posições alternativas. No geral concluiu que facilitava a monitorização à distância do intervalo QT, inclusive em doentes de ambulatório em quarentena sob terapêuticas com potencial para prolongamento do intervalo QT. ${ }^{6}$

Os estudos anteriormente referidos revelam a relação estreita entre a evolução tecnológica e o avanço da medicina. O desenvolvimento de dispositivos ou aplicações úteis, práticas e facilmente integradas no quotidiano podem aliar fitness, saúde e bem-estar com a monitorização do ritmo cardíaco, permitindo a deteção precoce de anomalias significativas que necessitem de eventual investigação e tratamento modificador de prognóstico adicional.

No entanto, a sua utilidade enquanto método de rastreio de FA é controversa, dada a sua especificidade e sensibilidade limitadas, bem como a sua custo-efetividade.

Refira-se ainda a problemática associada ao desenvolvimento tecnológico, e apontada por alguns autores: o sobrediagnóstico nos indivíduos notificados após deteção de pulso irregular apenas no smartwatch, conduzindo a uma marcha diagnóstica exaustiva, sobretratamento e ansiedade nos utilizadores. ${ }^{4}$

Apesar de a sua utilização apresentar algumas limitações como, por exemplo, o custo elevado e a adesão de grupos etários mais jovens, a tendência é para o aumento progressivo do número de utilizadores. No entanto, importa referir que os dispositivos e aplicações não substituem a avaliação clínica nem exames complementares de diagnóstico e, acima de tudo, não permitem efetuar diagnósticos. Podem, sim, alertar precocemente o clínico para determinado problema de saúde que, por sua vez, deve conduzir a uma investigação adicional, eventual diagnóstico e orientação clínica.

\section{AGRADECIMENTOS}

Os autores deixam um agradecimento especial à Dra Ana Sá, médica especialista em medicina geral e familiar, por todo o apoio no desenvolvimento do presente trabalho.

\section{REFERÊNCIAS BIBLIOGRÁFICAS}

1. Monteiro P. Estudo Safira: reflexões sobre a prevalência e os padrões de tratamento de fibrilhação auricular e risco cardiovascular em 7500 indivíduos com 65 ou mais anos [The SAFIRA study: A reflection on the prevalence and treatment patterns of atrial fibrillation and cardiovascular risk factors in 7500 elderly subjects]. Rev Port Cardiol. 2018;37(4): 307-13. Portuguese

2. Perez MV, Mahaffey KW, Hedlin H, Rumsfeld JS, Garcia A, Ferris T, et al. Large-scale assessment of a smartwatch to identify atrial fibrillation. N Engl J Med. 2019;381(20):1909-17.

3. Campion EW, Jarcho JA. Watched by Apple. N Engl J Med. 2019;381(20): 1964-5.

4. Qiu J, WangY.A smartwatch to identify atrial fibrillation. N Engl J Med. 2020;382(10):974-5.

5. U.S. Food \& Drug Administration. Electrocardiograph software for overthe-counter use [homepage]. FDA; 2018 [updated 2021 Sep 20]. Available from: https://www.accessdata.fda.gov/scripts/cdrh/cfdocs/cfpmn/ denovo.cfm?id=DEN180044

6. Strik M, Caillol T, Ramirez FD, Abu-Alrub S, Marchand H, Welte N, et al. Validating QT-interval measurement using the Apple watch ECG to enable remote monitoring during the COVID-19 pandemic. Circulation. 2020;142(4):416-8.

\section{CONFLITO DE INTERESSES}

Os autores declaram não possuir quaisquer conflitos de interesse.

\section{FINANCIAMENTO}

O trabalho que consta neste manuscrito não foi objeto de qualquer tipo de financiamento externo, incluindo bolsas de investigação.

\section{ENDEREÇO PARA CORRESPONDÊNCIA}

Carolina Araújo Soares

E-mail: carolinaaj.soares@gmail.com

https://orcid.org/0000-0002-4121-5249

Recebido em 16-12-2020

Aceite para publicação em 17-07-2021 


\section{ABSTRACT}

\section{FUTURE PERSPECTIVES IN CARDIAC RHYTHM MONITORING AND SMARTWATCH USE}

The regular use of smartwatches allows monitoring the exercise performed by its users, especially in terms of intensity, duration, energy expenditure, and more recently, heart rate. The potential use of these electronic devices in medicine is an important milestone, allowing through monitoring of cardiac rhythm, a possible way to detect periods of cardiac arrhythmias such as atrial fibrillation and more recently, QT interval prolongation that can cause potentially fatal ventricular arrhythmias, with its importance shown during COVID-19 pandemics. The main studies have several limitations; however, they show the importance of technological evolution and its potential prognostic impact on the users of these devices.

Keywords: Smartwatch; Monitoring; Remote sensing technologies; Atrial fibrillation; Cardiac arrhythmias. 